\title{
Infecciones osteo-articulares por Kingella kingae en niños en un hospital pediátrico de alta complejidad: epidemiología y factores asociados
}

\author{
Osteo-articular infections by Kingella kingae in children in a highly complex pediatric \\ hospital: epidemiology and associated factors
}

\author{
M. Guadalupe Pérez', Eva Verónica Deschutter², María Elena Venuta², Soledad Mussini, Adela Isasmendi², \\ Vanesa Reijtman², María Laura Tripodi³, Sofía Abel², Agustina Ponzone³, Alejandra Mastroianni², \\ José Luis Pinheiro², Eva García², Rosa Bologna y María Teresa Rosanova
}

\begin{abstract}
${ }^{1}$ Servicio de Control Epidemiológico e Infectología. Hospital de Pediatría Prof. Dr. Juan P. Garrahan. Buenos Aires, Argentina. ${ }^{2}$ Servicio de Microbiología. Hospital de Pediatría Prof. Dr. Juan P. Garrahan. Buenos Aires, Argentina.

${ }^{3}$ Servicio de Ortopedia y Traumatología. Hospital de Pediatría Prof. Dr. Juan P. Garrahan. Buenos Aires, Argentina.
\end{abstract}

Financiamiento: Este estudio no tuvo financiamiento.

Los autores no poseen conflictos de interés.

Recibido: 30 de octubre de 2019 / Aceptado: 12 de febrero de 2020

\section{Resumen}

Introducción: La epidemiología de las infecciones osteo-articulares (IOA) se ha modificado en los últimos años. La incidencia de Kingella kingae en Latinoamérica es desconocida. Objetivo: Describir la epidemiología de los niños con IOA. Estimar la incidencia de IOA causadas por K. kingae y compararlas con otras etiologías. Materialy Métodos: Cohorte prospectiva. Se incluyeron pacientes mayores de 1 mes de edad, hospitalizados entre el 1 de marzo de 2017 y 28 de febrero de 2019, con sospecha de IOA y procedimiento diagnóstico (biopsia o artrocentesis). Se utilizó STATA 13. Resultados: n: 84 pacientes. Se identificó la etiología en 58 pacientes $(69,1 \%)$. Predominaron Staphylococcus aureus (n: 44; 52,4\%) y K. kingae (n: 9; 10,8\%). En el período estudiado, la incidencia de IOA por $K$. kingae fue de 10,8 casos cada 100 IOA hospitalizadas. En el análisis multivariado, la edad inferior a 4 años (OR 13,8, IC95\% 5,5-82,7), el cuadro respiratorio reciente (OR 5,7, IC95\% 3,5-31,6, p 0,04) y la normalización antes del quinto día de la proteína C reactiva (PCR) (OR 3,8 IC95\% 1,8- 16,3, p 0,01$)$ se asociaron con las IOA por K. kingae. Conclusiones: En esta cohorte de niños, la incidencia de K. kingae fue de 10,8 casos cada 100 IOA. Kingella kingae representó la segunda etiología documentada, luego de $S$. aureus. La edad inferior a 4 años, el cuadro respiratorio reciente y la normalización antes del quinto día de la PCR cuantitativa se asociaron estadísticamente con IOA por K. kingae.

Palabras clave: Infección osteo-articular; artritis séptica; niños; Staphylococcus aureus, Kingella kingae.

\begin{abstract}
Background: The epidemiology of osteoarticular infections (IOA) has changed in recent years. The incidence of Kingella kingae in Latin America is unknown. Aims: To describe the epidemiology in patients with IOA in a children hospital. To estimate the incidence of IOA due to K. kingae and compare with other etiologies. Methods: Prospective cohort. Patients older than 1 month hospitalized between March, $1^{\text {th }}$ 2017 and February, $28^{\text {th }} 2019$ with suspected IOA and diagnostic procedure (biopsy or arthrocentesis) were included. STATA 13 was used. Results: $\mathrm{n}: 84$ patients. The etiology was identified in 58 patients (69.1\%). Staphylococus aureus predominated (n: 44; 52.4\%) and $K$. kingae (n: $9 ; 10.8 \%$ ). In the period studied, the incidence of IOA by $K$. kingae was 10.8 cases per 100 hospitalized IOA. In multivariate analysis, age less than 4 years (OR 13.8, 95\% CI 5.5-82.7), recent respiratory symptoms (OR 5.7, 95\% CI 3.5-31.6, p 0.04) and normalization before the fifth day of C-reactive protein (CRP) (OR 3.38 95\% CI 1.8-16.3, p 0.01 ) were associated with IOA by K. kingae. Conclusions: In this cohort of children the incidence of $K$. kingae was 10.8 cases per 100 IOA. Kingella kingae represented the second documented etiology, after $S$. aureus. Age under 4 years, recent respiratory symptoms and normalization before the fifth day of quantitative CRP were statistically associated with IOA by K. kingae.

Keywords: Osteoarthritis; septic arthritis; children; Staphylococcus aureus; Kingella kingae.
\end{abstract}




\section{Introducción}

L as infecciones osteo-articulares (IOA) representan una causa de morbilidad, internación y uso de antimicrobianos en pediatría. Su real incidencia en nuestro país es desconocida; en países desarrollados se reportan 22 casos cada 100.000 niños ${ }^{1}$. La mayoría de los casos se presenta en pacientes bajo 5 años de $\operatorname{edad}^{2} \mathrm{y}$ en varones ${ }^{3}$ No obstante, en los últimos años se describe un aumento en la mediana de la edad de presentación de IOA por Staphylococcus aureus resistente a meticilina $(\mathrm{SARM})^{3}$.

La principal causa identificada de IOA en niños es S. aureus $^{3,4}$. En las últimas décadas, $S$. aureus resistente a meticilina adquirido en la comunidad (SARM-AC) se ha distribuido mundialmente hasta alcanzar cifras epidémicas $^{3}$. En otras series, sin embargo, se han informado otros agentes etiológicos menos frecuentes como Streptococcus pneumoniae, Streptococcus pyogenes y Neisseria meningitidis ${ }^{4}$.

En años recientes, surgen descripciones de Kingella kingae como agente etiológico de IOA, sobre todo en niños en edad preescolar, con el antecedente de lesiones orales $^{5}$. Incluso, estudios realizados en niños identificaron a $K$. kingae como principal causa de $\mathrm{IOA}^{6}$.

En Latinoamérica, existen reportes de $K$. kingae en infecciones invasoras $\mathrm{y}$, especialmente, de IOA. Sin embargo, es un patógeno emergente que no se estudia en forma rutinaria.

Los objetivos de este estudio fueron: describir la epidemiología de los niños con IOA internados en el Hospital de Pediatría Prof. Dr. Juan P. Garrahan, Buenos Aires, Argentina, estimar la incidencia de IOA por $K$. kingae, describir sus características clínicas y comparar las IOA causadas por $K$. kingae vs otras etiologías.

\section{Material y Métodos}

Se realizó un estudio prospectivo de cohorte, incluyendo pacientes sobre 1 mes de edad y hasta 18 años, admitidos en el Hospital de Pediatría Prof. Dr. Juan P. Garrahan desde el 1 de marzo de 2017 al 28 de febrero de 2019 con sospecha de IOA, en quienes se efectuó un procedimiento diagnóstico (biopsia ósea o sinovial y/o artrocentesis). Se excluyeron los niños con antecedente reciente de internación, patología osteo-articular previa y/o inmunocompromiso, y los pacientes en quienes la evolución del cuadro clínico era mayor a 15 días.

Previo al inicio del estudio, se realizaron reuniones multidisciplinarias con los Servicios de Microbiología, Ortopedia y Traumatología, Infectología y Clínica Pediátrica, para difundir el modo correcto de referir las muestras de biopsia y/o artrocentesis, y poder incluir a todos los pacientes admitidos con sospecha de IOA en el período de estudio.

Se enrolaron en forma prospectiva los pacientes el día de su internación, se revisaron las historias clínicas y registraron antecedentes clínicos y los estudios de laboratorio realizados.

Fueron evaluados la edad, días de evolución previos a la consulta, tipo de IOA, examen físico, localización(es) de la(s) lesión(es), tipo y número de drenajes quirúrgicos (por artrocentesis, artrotomía o artroscopía), días de evolución al momento del drenaje y días de internación.

Se registraron variables de laboratorio, resultados de cultivos del líquido articular y de hemocultivos. También se determinó la sensibilidad in vitro a antimicrobianos de los agentes etiológicos involucrados.

Las muestras de hemocultivos y líquido articular o biopsia (ósea o sinovial) se procesaron en el laboratorio de Microbiología del hospital. Los hemocultivos fueron procesados mediante el sistema automatizado BacT/Alert $3 \mathrm{D}$ y posteriormente identificados mediante pruebas microbiológicas convencionales y automatizadas, siguiendo los protocolos de trabajo vigentes.

Las muestras de líquido articular y biopsia se sembraron en agar sangre, agar chocolate, agar cistina-lactosa deficiente en electrolitos (CLDE) y caldo tioglicolato, y fueron incubadas a $37^{\circ} \mathrm{C}$ por un período de 24 a $72 \mathrm{~h}$. La identificación bacteriana se llevó a cabo mediante MALDI-TOF MS (Vitek-MS, bioMérieux). Las pruebas de sensibilidad in vitro a los distintos antimicrobianos se realizaron por métodos automatizados (Vitek 2C, bioMérieux) y por el método de difusión con discos, según las normas del Clinical and Laboratory Standards Institute (CLSI) ${ }^{7}$.

En todos los casos se envió muestra de biopsia ósea y/o líquido sinovial para el diagnóstico molecular de $K$. kingae. Se empleó un método específico de reacción de polimerasa en cadena (RPC) en tiempo real "in house", de acuerdo al trabajo publicado por Cherkaoui A y cols. ${ }^{8}$. La técnica se optimizó en el laboratorio de Microbiología según las indicaciones de la bibliografia, con iniciadores dirigidos a las regiones $r t x A$ y $r t x B$ del locus RTX de KK. Para la extracción del ADN se utilizó el método comercial de QIAGEN (QIAamp DNA Mini Kit), siguiendo el protocolo de purificación de tejidos, según las instrucciones del fabricante. El extracto de ADN puro $(100 \mu \mathrm{l})$ se conservó a $-20{ }^{\circ} \mathrm{C}$ hasta la realización del ensayo. Se utilizó un equipo de detección ABI PRISM ${ }^{\mathrm{TM}} 7500$ de Applied Biosystems, con detección simultánea de dos fluoróforos diferentes (FAM para la secuencia blanco y VIC para el control interno de amplificación exógeno o IPC y para el gen constitutivo de $\beta$-globina). Para cada muestra se realizó la detección de los dos blancos específicos en forma independiente, además de un control de amplificación (IPC) como control de calidad de muestra. 
de K. kingae se realizó por RPC, excepto en un paciente en el que también desarrolló la bacteria en el cultivo de líquido sinovial.

Entre los 44 pacientes con identificación de $S$. aureus, $26(59,1 \%)$ tuvieron resistencia a meticilina y $18(40,9 \%)$ fueron sensibles. Se identificaron 5 aislados de $S$. aureus con resistencia a clindamicina $(11,4 \%)$.

En el período estudiado, la incidencia de IOA por $K$. kingae fue de 10,8 casos cada 100 IOA agudas hospitalizadas en la institución.

Evaluando las características de los pacientes en el grupo KK (n: 9, es 10,8\%) vs grupo no-KK (n: 75, es $89,2 \%)$ : la mediana de edad fue menor $(23,1$ meses vs $74,5, \mathrm{p}=0,023)$, se presentaron con mayor frecuencia como artritis (n: 6 , es $66,6 \%$ vs n: 33 , es $43,4 \%, p=0,30$ ) y la localización predominante fue en rodilla (n: 9 , es $100 \%$ vs $n: 23$, es $30,3 \% \mathrm{p}<0,001)$. En el grupo de pacientes con IOA por $K$. kingae, 8 tenían menos de 4 años $(88,9 \%)$ vs $30(40 \%)$ en el grupo no-KK.

Seis pacientes del grupo KK refirieron el antecedente de cuadro respiratorio en la última semana, $(66,7 \%)$ vs 9 en el grupo no-KK $(12 \%) \mathrm{p}<0,001$. La fiebre estaba presente en 6 pacientes $(66,6 \%)$ del grupo KK vs 62 del grupo no -KK (82,7\%). Ningún paciente en el grupo de IOA por $K$. kingae refirió el antecedente de traumatismo previo (Tabla 1).

La mediana de valor de proteína $\mathrm{C}$ reactiva (PCR) cuantitativa al ingreso fue 48,7 mg/L (RIC 37-50) en los pacientes con $K$. kingae vs 105,7 mg/L (RIC 32-150), $\mathrm{p}=0,01$. No hubo diferencias en el valor de eritrosedimentación al ingreso.

En el seguimiento, los pacientes con IOA por $K$. kingae requirieron menos días de hospitalización (5,6 vs 13,2; p 0,006). La PCR cuantitativa se normalizó mas precozmente (5,8 días vs 13,3 días, $\mathrm{p}=0,04)$; al quinto día de tratamiento, en 5 pacientes $(55,6 \%)$ del grupo KK vs 14 $(18,6 \%)$ del grupo no-KK, la PCR era normal, $p=0,01$.

En el análisis multivariado, ajustando por el resto de las variables: la edad inferior a 4 años (OR 13,8; IC95\% 5,5-82,7), el antecedente de cuadro respiratorio reciente (OR 5,7, IC95\% 3,5-31,6, p = 0,04) y la normalización antes del quinto día de la PCR cuantitativa (OR 3,38, IC95\% 1,8-16,3; $\mathrm{p}=0,01$ ) se asociaron con significación estadística con las IOA por K. kingae.

\section{Discusión}

Kingella kingae es un cocobacilo gramnegativo de difícil desarrollo en medios convencionales. En los últimos años, a partir del advenimiento de técnicas moleculares de diagnóstico, se identifica con más frecuencia como agente etiológico de bacteriemia, endocarditis e $\mathrm{IOA}^{9}$. Esta es una bacteria anaerobia facultativa, de difícil identifica- 


\begin{tabular}{|c|c|c|c|c|c|}
\hline Variable & \multicolumn{2}{|c|}{$\begin{array}{l}\text { Grupo Kingella kingae } \\
\text { n: } 9 \\
n \quad(\%)\end{array}$} & \multicolumn{2}{|c|}{$\begin{array}{l}\text { Grupo no-Kingella kingae } \\
\text { n: } 75 \\
n \quad(\%)\end{array}$} & p \\
\hline Sexo masculino & 5 & $(65,5)$ & 51 & (68) & 0,93 \\
\hline Mediana de edad en meses (RIC)* & \multicolumn{2}{|c|}{$23,1(13,5-28,5)$} & \multicolumn{2}{|c|}{$74,5(30-113)$} & 0,023 \\
\hline Enfermedad de base & 2 & $(22,2)$ & 15 & $(18,7)$ & 0,09 \\
\hline Menor de 4 años & 8 & $(88,9)$ & 30 & (40) & 0,001 \\
\hline Días de evolución hasta el diagnóstico & 6,9 & $(2-10)$ & 4,9 & $(2-7)$ & 0,3 \\
\hline Fiebre & 6 & $(66,7)$ & 62 & $(82,7)$ & 0,08 \\
\hline Días de fiebre. Mediana (RIC) & 3,7 & $(1-7)$ & 3,2 & $(1-4)$ & 0,71 \\
\hline Traumatismo & - & & 26 & $(34,6)$ & - \\
\hline Cuadro respiratorio previo & 5 & $(55,6)$ & 9 & (12) & 0,001 \\
\hline Escolarización & 2 & $(22,2)$ & 23 & $(30,6)$ & 0,57 \\
\hline Uso de antibióticos la semana previa a la internación & 3 & $(33,3)$ & 22 & $(29,3)$ & 0,6 \\
\hline Osteomielitis & - & & 18 & (24) & - \\
\hline Artritis & 6 & $(66,6)$ & 33 & (44) & 0,03 \\
\hline Osteoartritis & 3 & $(33,3)$ & 24 & (32) & 0,6 \\
\hline Localización rodilla & 9 & $(100)$ & 23 & $(30,3)$ & 0,001 \\
\hline Proteína C reactiva cuantitativa. Mediana UI/L (RIC) & 48,7 & $(37-50)$ & $105,7($ & 32-150) & 0,01 \\
\hline Eritrosedimentación, mm/h. Mediana (RIC) & 70,3 & $(49-102)$ & $73,2($ & $4-110)$ & 0,09 \\
\hline Días hasta la normalización de la proteína C reactiva & 5,8 & $(4-10)$ & 13,3 & $(7-17)$ & 0,04 \\
\hline Normalización de la proteína C reactiva al quinto día & 5 & $(55,6)$ & 14 & $(18,6)$ & 0,01 \\
\hline Días de internación & 5,6 & $(4-7)$ & 13,2 & $(6-15)$ & 0,006 \\
\hline
\end{tabular}

ción con los medios de cultivo que se utilizan de rutina. Ilharreborde y cols., reportaron la mejor sensibilidad del cultivo cuando los fluidos articulares se inoculaban sobre medios sólidos o cuando la muestra se colocaba en botellas de hemocultivos ${ }^{10}$. En pediatría, dado el escaso volumen del material obtenido del líquido sinovial, suele ser dificultoso el envío de múltiples muestras. A partir del uso de técnicas moleculares de diagnóstico, se ha registrado un aumento en la identificación de $K$. kingae como agente etiológico de las $\mathrm{IOA}^{5,11}$.

El diagnóstico adecuado permite estimar el pronóstico de las IOA y dirigir el tratamiento antimicrobiano, con la consiguiente disminución del uso de fármacos de amplio espectro.

En el estudio presentado, en coincidencia con otras publicaciones ${ }^{5}, K$. kingae representó el segundo agente etiológico de IOA, después de $S$. aureus.

En Latinoamérica, S. aureus se describe como el agente etiológico de mayor frecuencia en niños ${ }^{12}$. En la Argentina, en las últimas décadas, predomina SARM-AC. Un estudio previo realizado entre los años 2014 y 2016 en el
Hospital de Pediatría Juan P Garrahan, identificó SARMAC como principal agente etiológico de una cohorte de niños con IOA de similares características demográficas al estudio presentado ${ }^{3}$. Sin embargo, en el trabajo actual, S. aureus sensible a meticilina se identificó con más frecuencia que en el estudio anterior, aunque siguió predominando SARM-AC. En otros países de la región, como Uruguay, también se reportó esta disminución de la resistencia a meticilina en los últimos años ${ }^{12}$.

Kingella kingae coloniza la vía respiratoria de más de $70 \%$ de los niños a partir de los 6 meses y su frecuencia disminuye desde la edad escolar ${ }^{13}$. Por ello, los pacientes con menor edad tienen mayor riesgo de IOA por $\mathrm{K}$. kingae. La infección respiratoria alta, la estomatitis y otros factores que alteran la barrera mucosa suelen preceder a la enfermedad invasora por K. kingae. En un estudio que evaluó la presencia de virus respiratorios en pacientes con IOA, se observó que en $90 \%$ de los pacientes con $K$. kingae había co-infección con algún virus respiratorio, mientras que esto ocurrió sólo en $30 \%$ de los que tuvieron IOA por otra etiología ${ }^{13}$. 
en Argentina es clindamicina, dado que SARM-AC es el microorganismo que predomina ${ }^{20}$. Sin embargo, en pacientes bajo 4 años de edad, con cuadro respiratorio reciente y una mejor evolución, debe considerarse el agregado de $\beta$-lactámicos en el tratamiento definitivo.

Entre las fortalezas de este trabajo deben remarcarse que, para evitar sesgos en la estimación de la incidencia de las IOA por K. kingae, los pacientes se enrolaron en forma prospectiva durante dos años, involucrando en el trabajo a los médicos traumatólogos, infectólogos y microbiólogos. Se utilizaron criterios de exclusión para evitar el sesgo de selección de pacientes, ya que el estudio se desarrolló en un centro de alta complejidad, de derivación de niños con patologías complejas. El trabajo incluyó una cohorte de pacientes sin antecedentes de inmunosupresión ni cirugía osteoarticular previa, para mejorar la validez externa de los resultados.

\section{Conclusiones}

En esta cohorte de niños, la incidencia de $K$. kingae fue de 10,8 casos cada 100 IOA agudas. Kingella kingae representó el segundo agente etiológico en frecuencia, luego de $S$. aureus. La edad inferior a 4 años, el cuadro respiratorio reciente y la normalización antes del quinto día de la PCR cuantitativa se asociaron estadísticamente con IOA por $K$. kingae.

El uso de técnicas moleculares de diagnóstico optimizó la identificación de $K$. kingae como agente etiológico en estos pacientes.

\section{Referencias bibliográficas}

1.- Grammatico-Guillon L, Maakaroun Vermesse Z, Baron S, Gettner S, Rusch E, Bernard L. Paediatric bone and joint infections are more common in boys and toddlers: A national epidemiology study. Acta Paediatr 2013; 102 (3): e120-5. doi: 10.1111/apa.12115.

2.- Gafur O A, Copley L A, Hollmig S T, Browne R H, Thornton S A, Crawford S E. The impact of the current epidemiology of pediatric musculoskeletal infection on evaluation and treatment guidelines. J Pediatr Orthop 2008; 28 (7): 777-85. doi: 10.1097/ BPO.0b013e318186eb4b.

3.- Highton E, Pérez M G, Cedillo Villamagua C, Sormani M I, Mussini M S, Isasmendi A, et al. Infecciones osteoarticulares en un hospital pediátrico de alta complejidad: epidemiología y características clínicas asociadas con bacteriemia. Arch Argent Pediatr 2017; 116 (2): e204-e20. https://www.sap.org.ar/ docs/publicaciones/archivosarg/2018/ v116n2a11.pdf.
4.- Rosanova M T, Berberian G, Bologna R, Giménez S, Sarkis C, Buchovsky A, et al. Estudio descriptivo de infecciones osteo-articulares en niños en tiempos de Staphylococcus aureus resistente a meticilina de la comunidad (SARM-Co). Rev Chilena Infectol 2015; 32(3): 321-5. http://www.revista. sochinf.cl/Vol-32-3-2015/art11.pdf

5.- Chometon S, Benito Y, Chaker M, Boisset S, Ploton C, Bérard J, et al. Specific realtime polymerase chain reaction places Kingella kingae as the most common cause of osteoarticular infections in young children. Pediatr Infect Dis J 2007; 26 (5): 377-81. doi: 10.1097/01.inf.0000259954.88139.f4.

6.- Principi N, Esposito S. Kingella kingae infections in children. BMC Infect Dis 2015; 15: 260. https://doi.org/10.1186/s12879-0150986-9.

7.- Clinical and Laboratory Standards Institute. Performance Standards for Antimicrobial Susceptibility Testing; Twenty-Fifth Informational Supplement. CLSI document M100-S25. Wayne, PA: CLSI; 2015.
8.- Cherkaoui A, Ceroni D, Emonet S, Lefevre Y, Schrenzel J. Molecular diagnosis of Kingella kingae osteoarticular infections by specific realtime PCR assay. J Med Microbiol 2009; 58: 65-8. doi: 10.1099/jmm.0.47707-0.

9.- Paakkonen M, Peltola H. Bone and joint infections. Pediatr Clin North Am 2013; 60 (2): 425-36. doi: 10.1016/j.pcl.2012.12.006.

10.- Ilharreborde B, Bidet P, Lorrot M, Even J, Mariani-Kurkdjian P, Liguori S, et al. New real-time PCR-based method for Kingella kingae DNA detection: application to samples collected from 89 children with acute arthritis. J Clin Microbiol. 2009; 47: 1837-41.

11.- Gómez Bonduel V, Chayep D, Venuta M E, Hernández C, Casimir L, Pinheiro J L, et al. Utilización de un método molecular para la detección de Kingella kingae en muestras osteoarticulares. Revista de Medicina Infantil 2013; XX: 13-16. http://www.medicinainfantil. org.ar/images/stories/volumen/2013/xx_1_013. pdf.

12.- Zunino C, Vomero A, Pandolfo S, Gutiérrez C, Algorta G, Pírez M C, et al. Etiología y 
evolución de las infecciones osteo-articulares 2009-2015. Hospital Pediátrico del Centro Hospitalario Pereira Rossell, Uruguay. Rev Chilena Infectol 2017; 34 (3): 235-42. https:// scielo.conicyt.cl/pdf/rci/v34n3/art05.pdf.

13.- Basmaci R, Bonacorsi S, Ilharreborde B, Doit C, Lorrot M, Kahil M, et al. High respiratory virus oropharyngeal carriage rate during Kingella kingae osteoarticular infections in children. Future Microbiol 2015; 10 (1): 9-14. doi: $10.2217 / \mathrm{fmb} .14 .117$.

14.- Ceroni D, Cherkaoui A, Combescure C, Francois P, Kaelin A, Schrenzel J. Differentiating osteoarticular infections caused by Kingella kingae from those due to typical pathogens in young children. Pediatr Infect Dis J 2011; 30 (10): 906-9. doi: 10.1097/ INF.0b013e31821c3aee.
15.- Otero Reigada M C, Silveira L F, Policarpo S N, Pérez Tamarit M A, Martín A O, Durántez M S. Kingella kingae infections in the pediatric age. Enferm Infecc Microbiol Clin 2011; 29: 29-32. doi: 10.1016/S0213-005X(11)70024-6.

16.- Ceroni D, Cherkaoui A, Ferey S, Kaelin A, Schrenzel J. Kingella kingae osteoarticular infections in young children: clinical features and contribution of a new specific realtime PCR assay to the diagnosis. J Pediatr Orthop 2010; 30 (3): 301-4. doi: 10.1097/ BPO.0b013e3181d4732f.

17.- Basmaci R, Lorrot M, Bidet P, Doit C, Vitoux C, Penneçot G, et al. Comparison of clinical and biologic features of Kingella kingae and Staphylococcus aureus arthritis at initial evaluation. Pediatr Infect Dis J 2011; 30 (10): 902-4. doi: 10.1097/
INF.0b013e31821fe0f7.

18.- Alcobendas R, Remesal A, Murias S, Núñez E, Calvo C. Outpatients with acute osteoarticular infections had favourable outcomes when they received just oral antibiotics without intravenous antibiotics. Acta Paediatr 2018; 107 (10): 1792-7. doi: 10.1111/apa.14373.

19.- Yagupsky P, Katz O, Peled N. Antibiotic susceptibility of Kingella kingae isolates from respiratory carriers and patients with invasive infections. J Antimicrob Chemother 2001; 47: 191-3. doi: 10.1093/jac/47.2.191.

20.- Pérez M G, Rosanova M T. Infecciones osteoarticulares. Guías de atención pediátrica. 2019 Hospital Garrahan. [Acceso enero de 2020]. Disponible en: http://www.garrahan.gov. ar/images/intranet/guias_atencion/protocolo/ Infecciones_osteoarticulares.pdf. 